\title{
Lesiones toracolumbares en la práctica de acrobacia en tela. Reporte de casos
}

\author{
Pedro L. Bazán," Elio Marín,, Alejandro Betemps," Álvaro E. Borri," Martín Medina," Nicolás M. Ciccioli, \#\# Javier Reble” \\ "Unidad de Patología Espinal, Hospital Interzonal General de Agudos "General San Martín”, La Plata, Buenos Aires, Argentina \\ ** Departamento de Columna, Centro Médico de la Costa, Asunción, Paraguay \\ "Unidad de Columna Buenos Aires, Ciudad Autónoma de Buenos Aires, Argentina \\ \#Sección Columna, Clínica Pueyrredón, Mar del Plata, Buenos Aires, Argentina
}

\begin{abstract}
RESUMEN
Introducción: La acrobacia en tela es una práctica circense con muchos adeptos en la población urbana. Consiste en sostenerse en altura tomado por dos extremos de tela, para realizar posturas fijas y cambiar entre ellas mediante deslizamiento y caídas. La práctica profesional no está libre de lesiones. Objetivo: Conocer la naturaleza de la lesión, reconocer los factores predisponentes de lesión y las medidas de prevención utilizadas, evaluar la cinemática, clasificar la lesión y analizar el tratamiento. Materiales y Métodos: Se incluyeron seis pacientes. Se evaluaron la estructura física personal, la cinemática de la caída, los sistemas de protección, la clasificación de las fracturas según la nueva clasificación $\mathrm{AO}$, el cuadro neurológico, el tratamiento y las complicaciones. Resultados: La muestra incluyó a 6 mujeres, con un promedio de edad de 24 años y un índice de masa corporal de 19,29. Caída de $3,08 \mathrm{~m}$ de altura, cuatro con colchoneta $<5 \mathrm{~cm}$. Las lesiones principales fueron: 6 A1, 4 B2 y $1 \mathrm{C}$. No se observaron déficits neurológicos. Cuatro pacientes fueron sometidas a cirugía. Conclusiones: La práctica de acrobacia en tela es una actividad circense de reciente aparición global. Las mujeres están más expuestas a las lesiones. El entrenamiento riguroso y el empleo de elementos de seguridad son necesarios para evitar las lesiones. No se observó un patrón único de lesión, la causa fue multifactorial. El tratamiento indicado depende de la lesión vertebral.
\end{abstract}

Palabras clave: Lesión vertebral; columna toracolumbar; caída de altura; acrobacia en tela; práctica circense.

Nivel de videncia: IV

\section{Thoracolumbar spine injury associated with aerial silk practice. Case reports}

\section{ABSTRACT}

Introduction: Aerial silk is a circus practice that has become very popular in urban populations. It involves hanging from two pieces of fabric in order to make fixed positions and change between them by sliding and dropping from different heights. The professional practice is not free of injuries. Objective: To learn the nature of the injury, to recognize the injury predisposing factors and the safety measures used, to study the kinematics, to classify the injury, and to analyze the treatment. Materials and Methods: Six patients were included. The evaluation included individual body structure, drop kinematics, safety measures, classification of fractures according to the new AO classification, neurological symptoms, treatment, and complications. Results: The study included six female patients, averaging 24 years, a body mass index of 19.29 and a fall from $3.08 \mathrm{~m}, 4$ of which included mattress $<5 \mathrm{~cm}$. The main injuries classification resulted in $6 \mathrm{~A} 1,4 \mathrm{~B} 2$ and $1 \mathrm{C}$. No neurological deficit was found. Four patients underwent surgical treatment. Conclusions: Aerial silk is a circus activity that has recently become a worldwide practice. Women are more exposed. Rigorous training and the use of safety elements are necessary to avoid injuries. We did not observe a unique pattern of injury, because of its multifactorial cause. The therapeutic decision-making is related to the spinal injury.

Key words: Spinal injury; thoracolumbar spine; high fall; aerial silk; circus practice.

Nivel de Evidencia: IV

Recibido el 24-7-2019. Aceptado luego de la evaluación el 5-10-2019 • Dr. PEDRO L. BAZÁN • pedroluisbazan@ gmail.com

Cómo citar este artículo: Bazán PL, Marín E, Betemps A, Borri ÁE, Medina M, Ciccioli NM, Reble J. Lesiones toracolumbares en la práctica de acrobacia en tela. Reporte de casos. Rev Asoc Argent Ortop Traumatol 2020;85(2):119-124. https://doi.org/10.15417/issn. 1852-7434.2020.85.2.1010 


\section{INTRODUCCIÓN}

La acrobacia en tela, también conocida como tela aérea vertical (Figura 1), danza aérea, entre otras, es considerada una práctica circense que se ha desarrollado en los últimos años. El participante trabaja en altura tomándose de una tela de entre 14 y 20 m fijada a una estructura firme que forma dos telas de 7 a $10 \mathrm{~m}$ cada una. La práctica consiste en realizar distintas figuras fijas y dinámicas (Figura 2), desde las cuales se transitan mediante acrobacias conocidas como "caída" y "deslizada". Cada una de ellas requiere un uso distinto de la tela (juntas-separadas, tensas-libres, atadas-sueltas), las manos, el tronco y los pies. Como en cualquier otra actividad, su entrenamiento y su práctica pueden dividirse en inicial, intermedio y avanzado, según la intensidad de los movimientos y las figuras. ${ }^{1,2}$ Durante el entrenamiento, se utilizan elementos de protección sobre el piso que son colchonetas de distintas alturas. La práctica circense aérea se relaciona con una tasa de lesión de 7-9/1000, la columna y la rodilla son segmentos muy expuestos a sufrir lesiones (razón de probabilidades [odds ratio], 0,67 para la región toracolumbar), aunque la mayoría son leves, y la tasa de lesiones sin diagnóstico inicial es alta. ${ }^{3,4}$

Un estudio de la cinemática de las lesiones vertebrales mostró que las lesiones más graves se asociaban con caídas de más de $6 \mathrm{~m}$ de altura. ${ }^{5}$ La superficie de aterrizaje en las caídas juega un rol importante, ya que las superficies que producen un impacto corto, como el concreto, aumentan los grados de lesiones comparadas con superficies, como la nieve, el barro o la arena. ${ }^{6-8}$ Snyder, en un estudio de 128 pacientes que sufrieron caídas de altura, halló que la posición del cuerpo en el momento del impacto influye directamente sobre la naturaleza y la extensión de las lesiones; la posición de "primero pies" es la más frecuente y provoca una carga axial que lesiona huesos de grandes extremidades, la columna y la pelvis. ${ }^{7}$ Las lesiones de la columna espinal en los pacientes caídos de altura pueden pasar inadvertidas, sobre todo en aquellos que presentan traumatismos múltiples sin déficit neurológico. ${ }^{69,10}$

Los objetivos de este estudio fueron conocer la naturaleza y los factores predisponentes de la lesión, evaluar las medidas de prevención utilizadas, analizar la cinemática, clasificar la lesión y considerar las posibilidades de tratamiento.

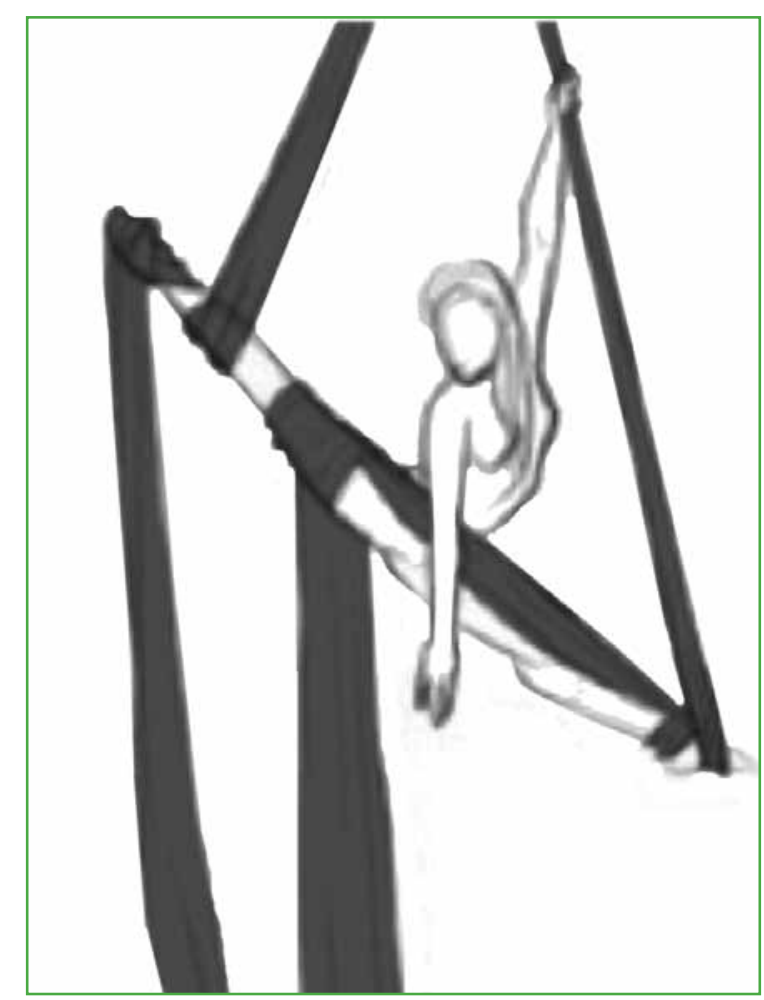

Figura 1. Imagen que muestra la utilización de piezas de tela para sujetarse en altura. 


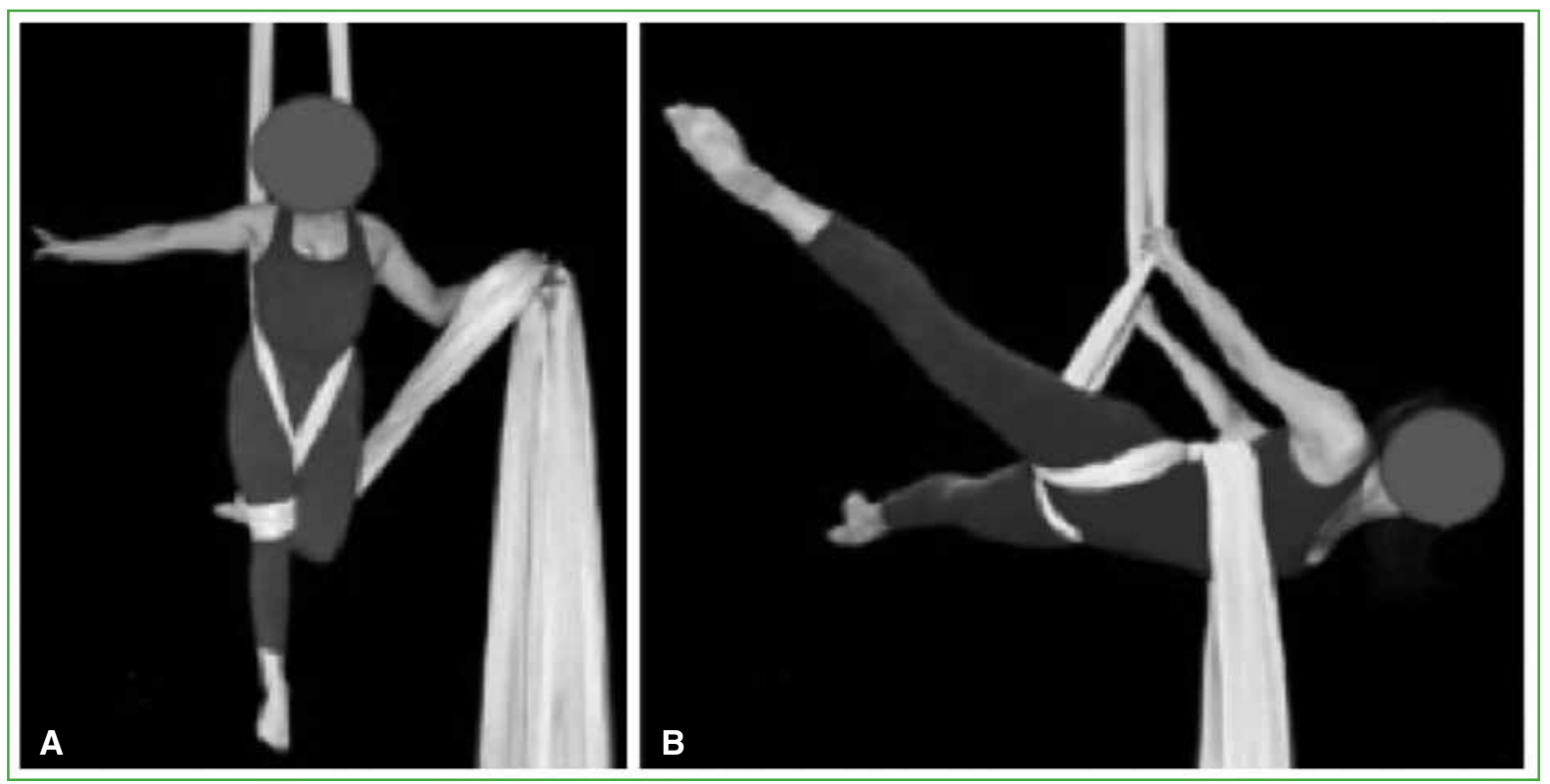

Figura 2. Imágenes que muestran las figuras llamadas "deslizada" (A) y "caída” (B). Tomado de Diaz Collao D, Reyes Camacho N, Sepúlveda Fuentes S. Metodología para la enseñanza de tela aérea vertical.

\section{MATERIALES Y MÉTODOS}

Estudio descriptivo observacional, retrospectivo multicéntrico, sobre una población de seis pacientes que ingresaron con diagnóstico de fractura vertebral ocurrida durante la práctica de acrobacia en tela.

Se evaluaron la estructura física personal, la cinemática de la caída, los sistemas de protección, la clasificación de las fracturas según la nueva clasificación $\mathrm{AO},{ }^{11}$ el cuadro neurológico, el tratamiento y las complicaciones.

\section{RESULTADOS}

La muestra incluye a seis mujeres, con un promedio de edad de 24 años (rango 15-36) y un índice de masa corporal promedio de 19,29 (peso normal) (Tabla). Todas tenían lesiones relacionadas con una caída de altura desde un promedio de 3,08 $\mathrm{m}$ (rango 1-5), durante la práctica recreativa de tela aérea vertical, en su entrenamiento controlado por un profesor. Cuatro pacientes utilizaban como sistema de protección una colchoneta de menos de $5 \mathrm{~cm}$ de alto. En un caso, se demoró cuatro días en llegar al diagnóstico.

Las lesiones vertebrales se localizaban en la columna torácica baja y la columna toracolumbar. Tres pacientes tenían más de una fractura vertebral (casos 1, 3 y 4). Se distinguieron seis fracturas con compresión axial y compromiso de un solo platillo, y sin compromiso del muro posterior (A1); cuatro lesiones de la banda posterior con compromiso ligamentario (B2) y otro caso con traslación en el plano sagital (C); tres lesiones B2 y la lesión C se asociaron con lesión secundaria A1 y la restante lesión B2, con una lesión por estallido completo. Ninguna paciente tenía déficit neurológico.

No se halló una relación directa entre el aumento de la altura de la caída y la gravedad o la complejidad de la lesión vertebral.

A dos pacientes se les indicó tratamiento no quirúrgico (casos 4 y 5) y cuatro fueron sometidas a cirugía por vía posterior (casos 1, 2, 3 y 6), según la decisión del médico tratante, en relación con las lesiones B2 y C. El tratamiento quirúrgico consistió en una estabilización instrumentada mediante tornillos pediculares y barras, asociado a la colocación de injerto óseo autólogo.

No se observaron complicaciones directas ni indirectas relacionadas con la terapéutica aplicada. Las pacientes retornaron a su vida normal en tiempos adecuados. Tres reanudaron la práctica de la acrobacia en tela. 
Tabla. Datos clínicos de las pacientes

\begin{tabular}{|c|c|c|c|c|c|c|c|c|c|c|c|}
\hline Paciente & Sexo & Edad & Talla & $\begin{array}{l}\text { Peso } \\
\text { (kg) }\end{array}$ & IMC & $\begin{array}{c}\text { Caída } \\
\text { (m) }\end{array}$ & Protección & Demora & ASIA & Lesión & Tratamiento \\
\hline 1 & $\mathrm{~F}$ & 22 & 1,65 & 50 & 18,37 & 1,5 & No & No & E & $\begin{array}{l}\text { L1-L2 B2 (A4) } \\
\text { T11-T12 B2 (A1) }\end{array}$ & Quirúrgico \\
\hline 2 & $\mathrm{~F}$ & 20 & 1,64 & 56 & 20,82 & 3 & $\begin{array}{l}\text { Colchoneta } \\
<5 \mathrm{~cm}\end{array}$ & No & E & T12-L1 B2 & Quirúrgico \\
\hline 3 & $\mathrm{~F}$ & 15 & 1,58 & 45 & 18,03 & 5 & No & No & $\mathrm{E}$ & $\begin{array}{l}\text { T10-T11 B2 } \\
\text { (T11 y L1 A1) }\end{array}$ & Quirúrgico \\
\hline 4 & $\mathrm{~F}$ & 33 & 1,68 & 52 & 18,42 & 1 & $\begin{array}{l}\text { Colchoneta } \\
<5 \mathrm{~cm}\end{array}$ & No & $\mathrm{E}$ & $\begin{array}{c}\mathrm{T} 2, \mathrm{~T} 3, \mathrm{~T} 4, \mathrm{~T} 5 \\
\text { y T6 A1 }\end{array}$ & No quirúrgico \\
\hline 5 & $\mathrm{~F}$ & 16 & 1,61 & 48 & 18,52 & 4 & $\begin{array}{l}\text { Colchoneta } \\
<5 \mathrm{~cm}\end{array}$ & 4 días & E & L1A1 & No quirúrgico \\
\hline 6 & $\mathrm{~F}$ & 36 & 1,64 & 58 & 21,56 & 4 & $\begin{array}{l}\text { Colchoneta } \\
<5 \mathrm{~cm}\end{array}$ & No & $\mathrm{E}$ & $\begin{array}{l}\mathrm{T} 11-\mathrm{T} 12 \\
\mathrm{C}(\mathrm{L} 1 \mathrm{~A} 1)\end{array}$ & Quirúrgico \\
\hline
\end{tabular}

$\mathrm{F}=$ femenino, $\mathrm{IMC}=$ índice de masa corporal, ASIA = American Spinal Injury Association.

\section{DISCUSIÓN}

La acrobacia en tela o tela acrobática es una de las más nuevas categorías de acrobacia aérea. En el circo, la expresión Acrobacia Aérea hace referencia a la disciplina practicada con instrumentos que cuelgan del techo. Otras categorías también conocidas incluyen: el trapecio, la lira y la danza vertical. ${ }^{11,12} \mathrm{Su}$ origen se remonta a alrededor de 1959, en la escuela de circos de Francia, donde el estudiante presenta su acto usando una o dos largas piezas de tela. Esta práctica se reconoce oficialmente desde 1998. ${ }^{11,13}$

El arte de la tela acrobática se difunde ampliamente desde 1998, gracias a Isabelle Vaudelle e Isabelle Chasse, durante la presentación llamada Quidam como parte del espectáculo del Cirque du Soleil. ${ }^{14}$ Desde mediados de la década de 1990, equipos de acróbatas británicos y franceses se interesaron en esta espectacular atracción y pronto se hizo popular en el resto del mundo. En 2002, la BBC emitió un programa de coreografías de acrobacias en telas durante 5 años. ${ }^{14}$

Sin embargo, hay un grupo que argumenta que el creador de esta acrobacia fue Andre Simard, en 1987, también como parte de nuevas presentaciones del Cirque du Soleil. ${ }^{14}$

En general, quienes practican esta disciplina pueden ser considerados tanto atletas como artistas. Su entrenamiento está asociado a altas demandas de esfuerzo físico con posturas y movimientos extremos. ${ }^{15,16} \mathrm{El}$ riesgo de lesiones serias en estudiantes circenses (la mayoría adolescentes) es del 2,8\%.2

Sobre la base de un estudio epidemiológico retrospectivo de los accidentes sufridos durante el entrenamiento de 169 estudiantes de entre 11 y 22 años de una escuela circense, se comunicaron un 28,3\% de lesiones espinales $(17,2 \%$ cervicales y $10,9 \%$ lumbares $) .^{2}$ Llamativamente no se reportaron lesiones torácicas, a diferencia de los casos incluidos en este estudio.

Las lesiones vertebrales se observan en acróbatas y en estudiantes, su gravedad depende de la altura y la forma de la caída, como así también de la prevención de las lesiones con la formación de los acróbatas y el uso de elementos de protección. ${ }^{7}$

Las lesiones pueden pasar inadvertidas cuando no se evalúa correctamente a los pacientes con antecedentes traumáticos y dolor. ${ }^{5}$ El tipo de tratamiento depende del cuadro neurológico y la lesión ósea o ligamentaria. 


\section{CONCLUSIONES}

La práctica de acrobacia en tela es una actividad circense de reciente implementación global. Las mujeres están más expuestas, porque son las que más practican esta actividad. El entrenamiento riguroso y el uso de elementos de seguridad son necesarios para evitar las lesiones que, al no provocar síntomas neurológicos, pueden no ser diagnosticadas inicialmente. No observamos un patrón único de lesión, la causa es multifactorial (altura de la caída, entrenamiento y ejercicio realizado). La decisión terapéutica está relacionada con la lesión vertebral.

Conflicto de intereses: Los autores no declaran conflictos de intereses.

ORCID de E. Marín: http://orcid.org/0000-0001-6204-8788

ORCID de A. Betemps: http://orcid.org/0000-0003-1586-9262

ORCID de Á. E. Borri: http:///orcid.org/0000-0002-5568-867X
ORCID de M. Medina: http://orcid.org/0000-0002-5281-5645

ORCID de N. M. Ciccioli: http://orcid.org/0000-0002-5851-2821

ORCID de J. Reble: http://orcid.org/0000-0003-4772-9715

\section{BIBLIOGRAFÍA}

1. Diaz Collao D, Reyes Camacho N, Sepúlveda Fuentes S. Metodología para la enseñanza de tela aérea vertical. www.saberesdecirco.com > uploads > 2018/12 > ensenanza_tela_aerea

2. Wanke EM, McCormack M, Koch F, Wanke A, Groneberg D. Acute injuries in student circus artists with regard to gender specific differences. Asian J Sports Med 2012;3(3):153-60. https://doi.org/10.5812/asjsm.34606

3. Wolfenden HEG, Angioi M. Musculoskeletal injury profile of circus artists. A systematic review of the literature. Med Probl Perform Art 2017;32(1):51-9. https://doi.org/10.21091/mppa.2017.1008

4. Shrier I, Meeuwisse WH, Matheson GO, Wingfield K, Steele RJ, Prince F, et al. Injury patterns and injury rates in the circus arts. An analysis of 5 years of data from Cirque du Soleil. Am J Sports Med 2009;37(6):1143-8. https://doi.org/10.1177/0363546508331138

5. Bazán PL, Borri AE, Feldman D, Szmidt VE. Evaluación cinemática del trauma raquimedular. Presentado en formato Póster Electrónico durante el IX Congreso SILACO - XXI Congreso GEER, Santander, España, 31 de mayo, 1 y 2 de junio de 2007.

6. Bazán PL, Borri AE, Szmidt VE. Lesiones vertebrales y medulares en pacientes que presentaron caídas de altura. Rev Asoc Platense Ortop Traumatol 2006/2009:149-154.

7. Sawyer JR. Fracture patterns in children and young adults who fall from significant heights. J Pediatr Orthop 2000;20(2):197-202. PMID: 10739282

8. Goodacre S, Goyder E, Joseph A. Can the distance fallen predict serious injury after a fall from a height? J Trauma 1999;46(6):1055-8. https://doi.org/10.1097/00005373-199906000-00014

9. Wittenberg R, Hargus S, Reinhard S. Noncontiguous unstable spine fractures. Spine (Phila PA 1976) 2002;27(3):254-7. https://doi.org/10.1097/00007632-200202010-00010

10. Hamilton GM, Meeuwisse WH, Emery CA, Shrier I. Examining the effect of the injury definition on risk factor analysis in circus artists. Scand J Med Sci Sports 2012;22(3):1-5. https://doi.org/10.1111/j.1600-0838.2010.01245.x

11. Vaccaro AR, Oner C, Kepler CK, Dvorak M, Schnake K, Bellabarba C, et al. AOSpine Thoracolumbar Spine Injury Classification System. Fracture description, neurological status, and key modifiers. Spine (Phila Pa 1976) 2013;38(23):2028-37. https://doi.org/10.1097/BRS.0b013e3182a8a381

12. Löber M. Ergebnisse der Untersuchung zur beruflichen Beanspruchungssituation von Artisten: Untersuchungsbericht. Arbeitsmedizinische Informationen für Theater und Orchester 1985;1:18. 
13. Loock F, Windisch B, Katzschke N. Rahmenarbeitsplatzcharakteristiken. Arbeitsmedizinische Informationen für Theater und Orchester 1982;1:12.

14. www.cirquedusoleil.com/en/jobs/casting/team/mentor/andre-sinard.aspx

15. Kahle S. Probleme der Arbeitshygiene und Berufskrankheiten bei Artist/innen. En: Bemmann H. Red. (ed). Die Artisten. ihre Arbeit und ihre Kunst. Berlin: Henschel; 1970:213-36.

16. Solomon R, Brown T, Gerbino PG, Micheli LJ. The young dancer. Clin Sports Med 2000;19(4):717-39. https://doi.org/10.1016/s0278-5919(05)70234-9 